\title{
Sarcoma Care Practice in India During COVID Pandemic: A Nationwide Survey
}

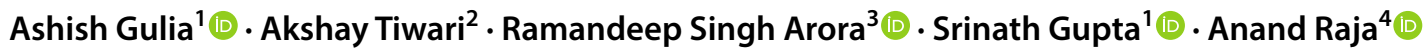

Received: 3 June 2020 / Accepted: 17 July 2020 / Published online: 31 July 2020

(c) Indian Orthopaedics Association 2020

\begin{abstract}
Background Amidst the COVID-19 pandemic, management of cancer has been one of the most intensely debated topics across the globe. We conducted an online survey to determine the consistency/or the lack of it, in the management of sarcoma patients between centres and the changes in policies.

Methods A twenty-five question online survey was conducted among practicing physicians over a period of 10 days using online portal (surveymonkey.com). It was followed by a critical analysis based on responses to each question.

Results Of 194 medical professionals who participated, 80\% were surgeons and 53\% were working in government institutes. Most respondents (81\%) continued their practice with some modifications. In OP majority $(67 \%)$ relied only on symptom, contact enquiry and temperature recording for screening. COVID-19 testing was done more (43\%) in IP patients. Most of institutes $(83 \%)$ followed rotational policy to reduce the number of staff at risk while $57 \%$ offered an alternate accommodation. $52.3 \%$ continued chemotherapy for all patients while radiotherapy for all was offered by $45 \%$. In metastatic cases, majority preferred either no treatment or non-surgical intervention (71\%).84.5\% believed in adapting changes (42\%-avoid supra major surgeries, $27 \%$-Operating only emergency cases and $15.5 \%$-High grade sarcomas with curative intent) in surgical management of sarcomas. For benign bone tumors, majority ( $71 \%$ ) agreed on adapting changes while $25 \%$ agreed on deferring all cases. 69\% preferred teleconsultations for follow-up. Complete PPE were being used for all aerosol generating procedures by $44 \%$. Only two thirds agreed with their institutes policy of PPE usage and COVID-19 testing.

Conclusion This survey has highlighted disparity on COVID-19 screening and management in various institutes across the country. This will act as a reference point for tracking future trends in bone and soft tissue tumor management guidelines, as the COVID-19 scenario unfolds globally and particularly in India.
\end{abstract}

Keywords COVID19 $\cdot$ Sarcoma $\cdot$ Survey $\cdot$ PPE $\cdot$ India

\section{Introduction}

Amidst the COVID-19 pandemic, management of cancer has been one of the most intensely debated topics across the globe $[1,2]$. Most of the attention, resources and priorities of

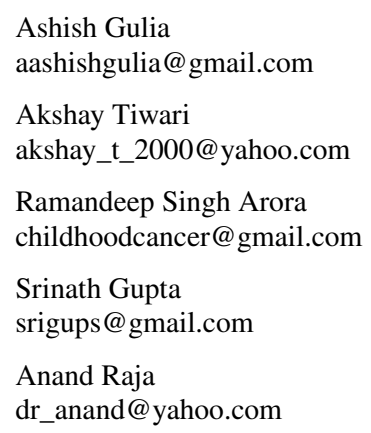

the healthcare systems have been directed towards containing the pandemic. This, coupled with delayed presentation of patients (both from fear of going out and from a lack of means of travel during the lockdown) is likely to lead to a delayed diagnosis and stage migration of a large number

1 Dept of Surgical Oncology, Tata Memorial Hospital, and Homi Bhabha National Institure (HBNI), Mumbai, India

2 Musculoskeletal Oncology, Max Institute of Cancer Care, Saket, New Delhi, India

3 Paediatric Oncology, Max Super Speciality Hospital, New Delhi, India

4 Department of Surgical Oncology, Cancer Institute (WIA), Adyar, Chennai, India 
of cancer patients [3]. Even for patients who do manage to reach the right centre, prioritising their treatment while containing COVID-19 transmission among both the healthcare staff and patients themselves has been a difficult balancing act for oncologists across the world [4]. This becomes particularly relevant given that healthcare workers and cancer patients are a higher risk group for morbidity and mortality due to COVID-19 infection [4, 5].

Management of bone and soft tissue sarcomas is no exception to this dilemma [6]. Delay in the treatment of these rare cancers is a threat to both limb and life, and there is no consensus on how to prioritise their management in the times of this pandemic. In the presence of ever evolving guidelines that change within and between countries, the pattern of care offered for bone and soft tissue sarcomas is bound to vary between centres. We conducted a survey amongst oncologists across India who are involved in the management of bone and soft tissue sarcomas, to analyse the patterns of screening, prioritising, and managing these patients within the constraints of resources and policies available with them.

The purpose of this survey was to determine the consistency, or the lack of it, in the patterns of care offered to sarcoma patients between centres and the policies followed. This would provide a much-needed insight of how the management of bone and soft tissue sarcomas is going to unfold in the coming times and beyond. A valuable outcome of this survey would be to formulate future strategies in the management of sarcomas in India, suggest ways to remove bottlenecks and propose modifications in sarcoma management in COVID times. These precious inputs by sarcoma experts across India could also form the basis of research looking at modifications in the diagnosis, management and follow-up of these patients even beyond the COVID-19 pandemic, having been tested in COVID times.

\section{Materials and methods}

\section{Survey Design and Content}

The author group conducted a comprehensive literature review on available data and practices in management of musculoskeletal tumors. A 24-item questionnaire was developed after deliberation within the study team (Table 1). This survey covered three main domains (a) participants speciality and working environment (b) Institutes' policies related general COVID-19 prevention and management (c) practices related to musculoskeletal tumor management in COVID-19 pandemic. The electronic version was designed and created

Table 1 Questionnaire on management of musculoskeletal tumors in COVID-19 pandemic

1. What is your specialty?

2. Your work setup is

3. What is the incidence of COVID-19 in your state?

4. How will you describe your practice during lockdown?

5. How are you/your institution screening your OPD patients for COVID-19?

6. How has your policy changed during the COVID-19 pandemic for OP?

7. How are you/your institution screening your IP patients for COVID-19?

8. Do you agree with current COVID-19 testing policies at your institution?

9. If "NO", what should be the testing policy?

10. Have you incorporated any change in staffing pattern at your workplace?

11. Have staff been given an alternative accommodation option in case they do not desire to return home?

12. What is your policy on chemotherapy for sarcomas during COVID-19 pandemic?

13. What is your policy on major surgeries for sarcomas during COVID-19 pandemic?

14. What is your policy on managing benign bone tumors during COVID-19 pandemic?

15. What is your policy on radiation therapy for sarcomas during COVID-19 pandemic?

16. How is complete PPE (jumpsuits, N95 mask, face shield, double gloves, shoe covers) being used in your setup?

17. If not using complete PPE, what is being used at your institution?

18. Do you agree with current policies for use of PPE at your institution?

19. If "NO", what should be the correct policy?

20. What is your current policy for routine follow-up of sarcoma patients during COVID-19 pandemic?

21. Are you using formal tele consults/virtual consults during COVID-19 pandemic?

22. What do you think about palliative treatment for sarcomas during COVID-19 pandemic?

23. How are you treating sarcoma with metastatic disease during COVID-19 pandemic

24. How long do you believe we will need to continue with the current pattern of working? 
using the SurveyMonkey platform (SurveyMonkey Inc. San Mateo, California, USA). The survey was piloted by seven experts, who were not from the study team (Fig. 1). This helped us to check the content, its relevance and validity.

\section{Survey Dissemination}

We conducted the survey over duration of 10 days from 21 st April 2020 to 30th April 2020. The survey was circulated to medical professionals involved in evaluation and management of Musculoskeletal Tumors. These included professionals from orthopedics, surgical oncology, medical oncology, radiation oncology and other allied fields such as anesthesiology, palliative medicine and physiotherapy. We utilized various online platforms for circulation of survey, these included emails, WhatsApp groups and Telegram messenger services. The introductory note explained the purpose of our survey. The embedded link helped participants to take the online survey via the website of the survey platform. Two reminders were staged on day 5 and day 8 of survey. To further increase the sample size, we posted the survey link during our online classes. The data collection was closed on 30th April, 2020 and was analyzed. The results were collated, and descriptive analysis was done.

\section{Results}

\section{Description of Respondents}

A total of 194 medical professionals took part in the survey. These were $52 \%$ orthopedic surgeons, $28 \%$ oncological surgeons, $5.1 \%$ medical oncologists, $2.6 \%$ radiation oncologists and the rest of them were anesthesiologists, pathologists, palliative medicine and physiotherapists. 53\% of the participants were working in government institutes, 33.5\% in hospitals in the private sector, and the remaining $13.5 \%$ belonged to non-teaching government institutes and selfowned nursing homes/clinics (Fig. 2). We have had respondents all across India (25 states including union territories) and 14 of them from international centres. Of these, 6 states had more than 1000 cases, 7 states had 200-1000 cases and
Fig. 1 Survey designing and content testing

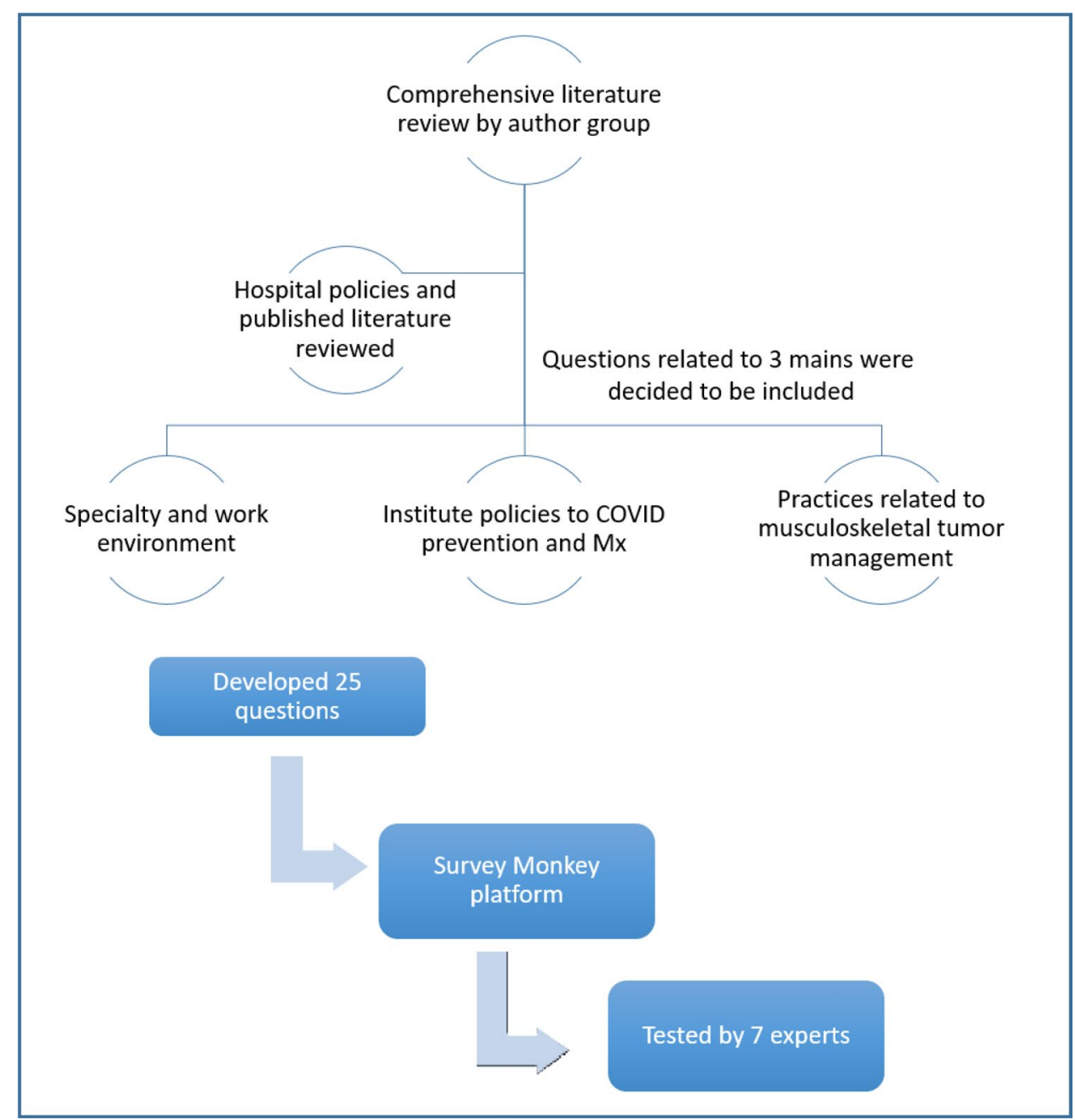


Fig. 2 Description of respondents and work environment

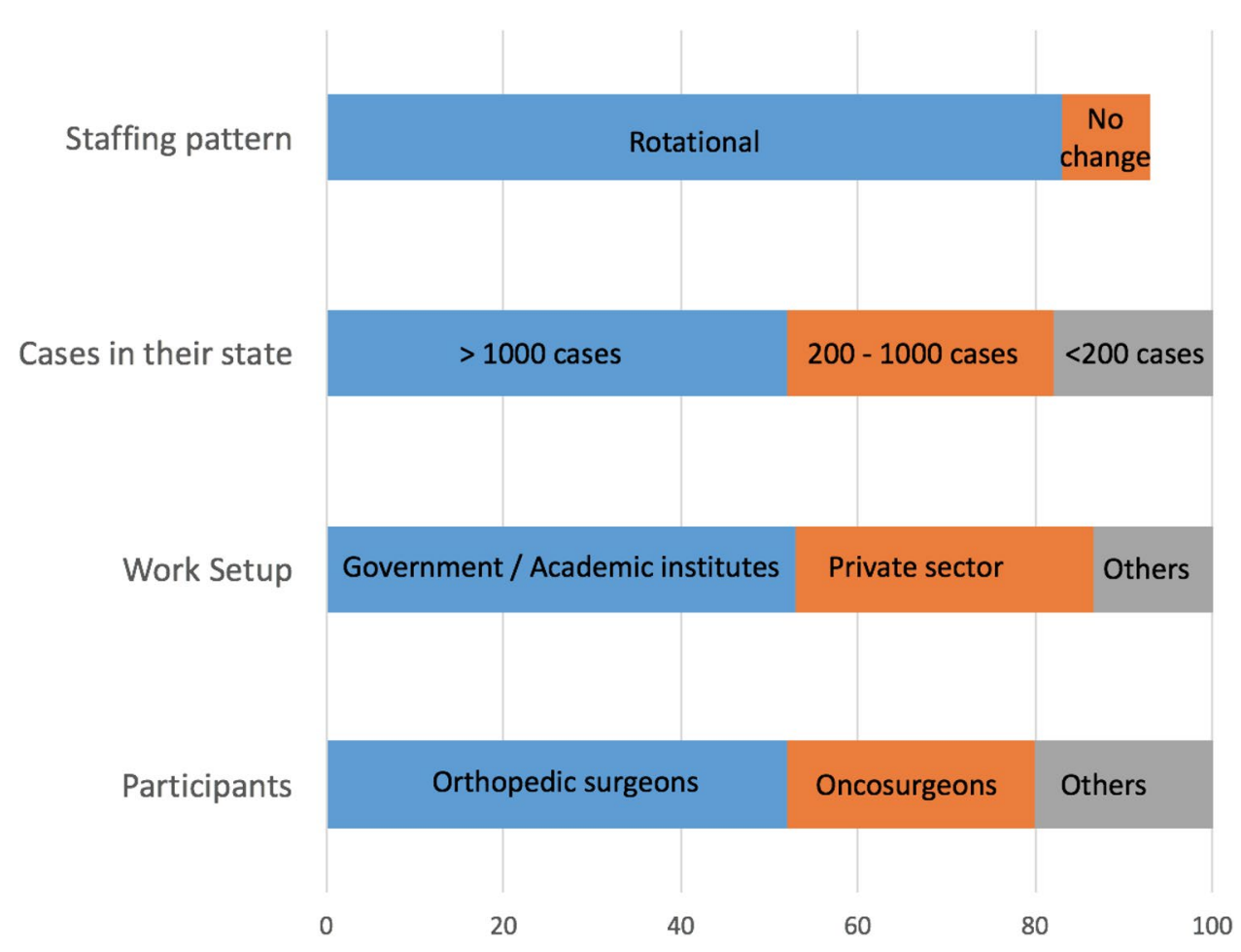

12 states had less than 200 cases the highest being Maharashtra, Delhi, Gujarat and Tamilnadu.

\section{Overall Changes in Practice during COVID-19}

Most respondents $(81 \%)$ were continuing their practice with some modifications, while in $10 \%$ practice was running as usual and in $9 \%$ there were no outpatient (OP) or interventional services (Fig. 3). The modifications included providing emergency services and OP to new patients only in $36 \%$, emergencies only in $33 \%$ and other modifications in the remaining (Fig. 3). In OP, $10 \%$ were screened by symptom and contact enquiry, $57 \%$ had additional temperature recording while 30\% also had COVID-19 testing (Fig. 3). In inpatient services (IP), the proportion being tested for COVID-19 was higher at $43 \%$ (Fig. 3). $38 \%$ of the participants disagreed with the current policies of their institutes. They were of the opinion of inclusion of COVID-19 testing for operative cases, inpatients $(31 \%)$ and those undergoing noninvasive procedures (14\%) while $27 \%$ felt it has to be
Fig. 3 Changes in OP/IP during COVID-19

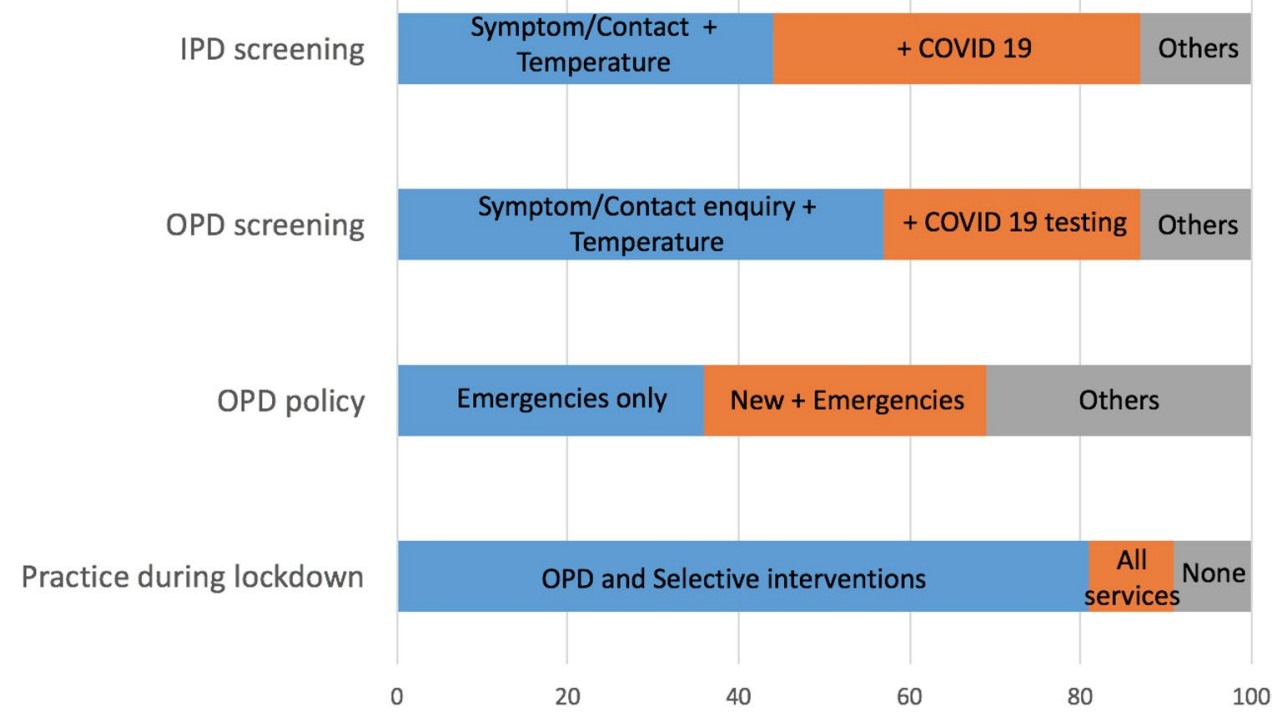


done for all patients visiting the hospital. $83 \%$ of them said that their institute was following rotational policy to reduce the number of staff at risk (Fig. 2). 57\% of them have been offered an alternate accommodation option.

For follow-up, $69 \%$ are doing teleconsultations via phone and advising patients to come back after the restrictions subside, $14 \%$ were continuing follow-up (FU) as usual while $11 \%$ had ceased all FU (Fig. 4). 20\% believed this practice teleconsultations will continue even after the pandemic while $60 \%$ were going to end it after the pandemic (Fig. 4). $32 \%$ believed the current restrictions will continue for 2 more months while $29 \%$ were of the opinion the current pattern of work is essential for the next 6 months.

\section{Changes in Sarcoma-Directed Curative and Palliative Treatment During COVID-19}

$52.3 \%$ said they were continuing chemotherapy for all patients while $31 \%$ said they were not enrolling new patients while continuing chemotherapy for those who are already receiving it, in $5.7 \%$ all chemotherapy delivery had been discontinued and with modifications in $11 \%$ (Fig. 5). Specifically, for patients with metastatic disease, $28 \%$ offered only best supportive care, $37 \%$ offered sarcoma-directed treatment but preferred stereotactic body radiation therapy (SBRT) over surgery for pulmonary metastasis. Curative treatment including pulmonary metastectomy for select group of patients was being offered only in $29 \%$.

Surgical management of sarcomas was as per usual only in $15.5 \%$ of respondents, with the rest adapting, including $42 \%$ who were avoiding procedures requiring long duration and blood loss, $27 \%$ were operating only emergency cases, while in $15.5 \%$ surgery was done only in high grade sarcomas with curative intent (Fig. 5). In contrast for benign bone tumors, surgical management of benign bone tumors was as per usual only in $3 \%$ of respondents, with the rest adapting (41\% operate only for surgery, $20 \%$ for locally aggressive lesions, $11 \%$ others) and in $25 \%$ surgeries were completely deferred (Fig. 5). 45\% were administering radiotherapy for all sarcoma cases as indicated, while $23 \%$ were not taking up new patients and $20 \%$ were administering it only in emergency and definitive treatment scenarios (Fig. 5).

For those with palliative care need, only $13 \%$ were offering usual palliative care service. Modified practice included home-based care only in 27\%, OP-based oral medication only in $9 \%$, and chemotherapy and/or radiotherapy but not surgical treatment in the remaining.
Fig. 4 Results - Use of PPE and follow-up consultations

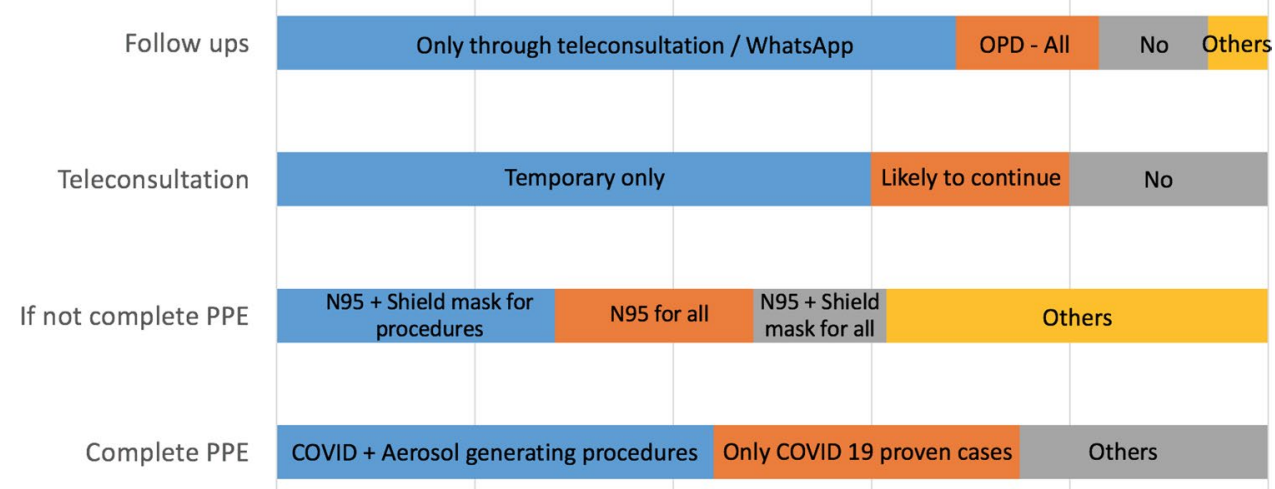

Fig. 5 Changes in sarcomadirected treatment during COVID-19

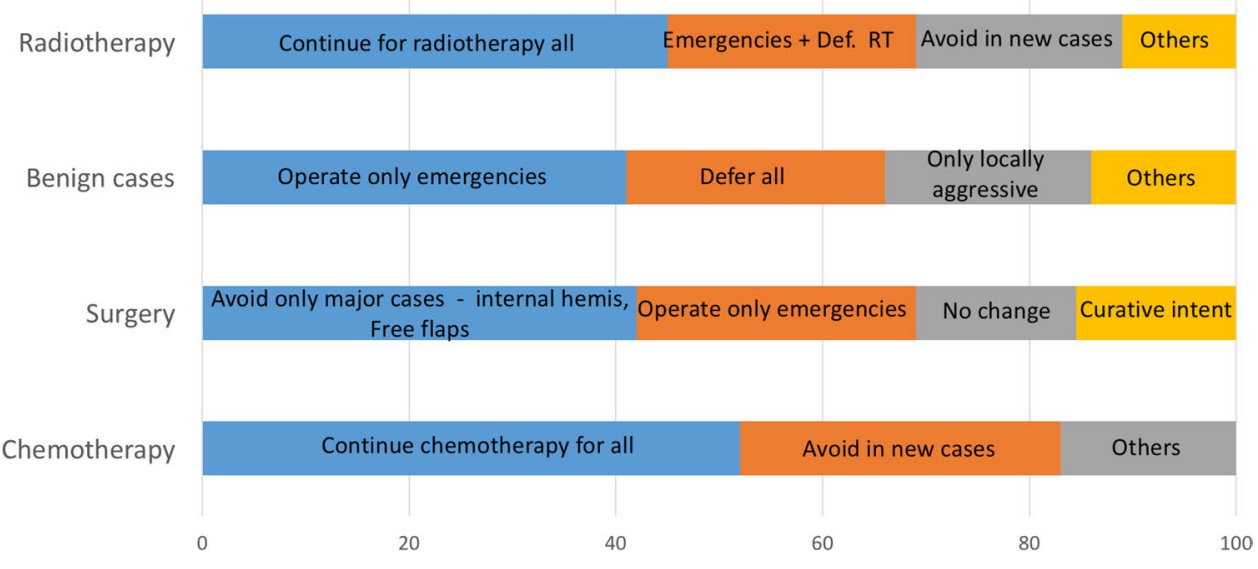




\section{Use of Personal Protective Equipment (PPE)}

Complete PPE (jumpsuits, N95 mask, face shield, double gloves, shoe covers) are being used for only proven COVID19 patients in $31 \%$ of the institutes while $44 \%$ were using it for all aerosol generating procedures in addition to use during treating a known case of COVID-19 (Fig. 4). As an alternate to complete PPE, partial protection in the form of N95 masks were used in $20 \%$ of the institutes while N95 + face shields were used mandatorily during all interventional procedures in $28 \%$ (Fig. 4). Only two thirds agreed with their institutes' policy of PPE usage selection and supported the use of PPE for all non COVID-19 cases undergoing aerosol generating procedures.

\section{Discussion}

COVID-19 has been a pandemic with an unprecedented impact for several reasons. Firstly, it can spread and infect a large number of people and cause morbidity and mortaility in a small fraction. Secondly, it can affect healthcare providers thus interrupting delivery of healthcare workers. Lastly, the lockdown initiated by the COVID-19 pandemic as an intervention to slow the spread and prepare services, has had an unparalleled detrimental effect on patient's ability to access healthcare in India. These challenges become particularly relevant in the care of patients with cancer and specially sarcomas which require multidisplinary timely management to achieve optimal outcomes.

This survey was conducted to understand the delivery of care for sarcomas by oncologists across India. Surgeons are usually the first point of contact for sarcoma and hence were predominant (80\%) amongst respondents (Fig. 2).

The results of our survey highlighted significant disruptions in delivery of cancer care. COVID-19 has placed an enormous strain on medical facilities and most institutions have brought about dramatic changes in practice. Less than $10 \%$ of respondents have continued to deliver their practice with no changes. Most of them have adapted OP, IP and FU services while $9 \%$ have completely shut down delivery of sarcoma-directed services. There was encouraging use of $80 \%$ of respondents for tele consults, with $20 \%$ stating their intent to continued use beyond the pandemic (Fig. 4). This could become a study model to test its utility during non COVID-19 times.

Treatment of cancer is a delicate balance between preventing up-staging and avoiding attendant morbidity/mortality. Expectedly, majority of the respondents were of the opinion that emergencies and newly diagnosed cases should not be denied treatment for sarcoma, without compromising on the safety of health care workers ( $\mathrm{HCW})$, patients, and their attendants. This is in alignment with the limited opinions available in the literature $[6,7]$.

The French Sarcoma group issued recommendations that encourage maintaining neoadjuvant and adjuvant chemotherapy and radiotherapy for all patients who are not suspected to have a COVID-19 infection, and advised provisions for adequate post-operative resuscitation capacities for high-risk surgeries [6]. Similar guidelines have been published us keeping an Indian perspective in mind [7] but, approximately half the respondents in our survey stated that chemotherapy and radiotherapy were no longer available for new patients.

Radiation oncology clinics have unique issues [8]. Most patients are outpatients and need travel. Difficulties in isolating patients, shared machine time, pre-existing disease states and immunocompromised status add to the prospects of delivering safe and effective radiotherapy. Denial of treatment for patients already on treatment may wreak havoc with radiation treatment planning. Practices for delivery of radiation varied widely which can be a result of lack on clarity and consensus on ideal practices and we believe this will improve over a period.

Surgery forms the mainstay of sarcoma treatment. Respondents were sensitive to the risks of exposure and difficulty in operating prolonged surgeries with full PPE. 82 (42\%) respondents operated only on high grade sarcomas with curative intent, but avoid major surgeries like internal hemipelvectomies, free flaps, etc. and 52 respondents (27\%) operated only emergencies (Fig. 5). These policies are also likely to change/evolve over time as the availability of healthcare infrastructure and $\mathrm{HCW}$ improves. As benign bone conditions are not life-threatening, there was a broad consensus to defer elective surgery for these patients and operate only on patients with emergencies or locally aggressive lesions by 48 and 39 respondents, respectively. 22 respondents offered alternate non-surgical modalities (Fig. 5).

PPE is resource-constrained and rational use is obligatory. Ministry of Health and Family Welfare, Govt of India recommends full complement of PPE use by HCWs for (a) Intensive Care Unit (ICU)/critical care patients, (b) emergency severely ill patients of Severe Acute Respiratory Infection (SARI) (c) during transport of SARI patients (d) sample collection, transport and testing and (e) autopsy [9]. We found that there was wide variation in use of full PPE. The false-negative COVID-19 reports might be a reason for respondents to use full PPE even in patients who are negative for COVID-19. For respondents not using full PPE, components of full PPE were used in multiple combinations which differed widely. This heterogeneity might reflect PPE availability across institutes depending on resources.

Our survey highlights that staffing is a huge challenge. Availability of HCW is key in delivery of care. Rotational 
staffing policies ensure limitation of exposure and ensures continuity of care. 161 respondents $(83 \%)$ were following this policy. Isolation of $\mathrm{HCW}$ by providing alternative accommodation at hospitals is ideal to prevent both, exposure of $\mathrm{HCW}$ and exposure by $\mathrm{HCW}$ in the community. 83 respondents (43\%) were not provided alternate accommodation. $80 \%$ of our respondents anticipate that current working pattern might need to be extended between 2 and 6 months. In the absence of guidelines by societies and associations, it is upon the individuals to take the call and strike a balance between continuing care and ensuring safety as of now. Non-uniform availability of resources, differential hospital setups, multitude of "guidelines", limited hospital capacity, including ICU and lack of point-of-care testing and seroprevalence data adds to the difficulty [10]. We expect to have more robust policies in near future, as both the COVID-19 pandemic and the outcome of sarcoma care evolves over time.

The global approach to COVID-19 testing has been nonuniform [11]. The incidence of asymptomatic carriers is unknown and is reflected in differential screening patterns across Institutions. In our survey, non-uniform screening procedures were practiced. In OP, $10 \%$ were screened by symptom and contact enquiry, $57 \%$ had additional temperature recording while 30\% also had COVID-19 testing (Fig. 3). In IP, the proportion being tested for COVID19 was higher at $43 \%$ (Fig. 3). Not surprisingly, 38\% of respondents disagreed with their screening policy. Indian Council of Medical Research (ICMR) guidelines for testing for COVID-19 have evolved since the declaration of pandemic in March 2020 to May 2020 and that has impacted on indvidual hospitals practices of testing $[12,13]$.

Palliative care services are under-resourced at the best of times [14]. As health systems become strained under COVID-19, providing safe and effective palliative care, including end-of-life care, becomes especially vital and especially difficult. Palliative treatment was offered by 141 (73\%) respondents. Palliative surgery was generally not recommended in this survey. Pharmacological interventions/ radiation/chemotherapy were used alone or in combination. Metastatic disease though low on priority during triage were not ignored with 56 respondents $(29 \%)$ offering definite curative therapy including metastasectomy for select group of patients with good prognosis (oligometastatic disease) and 71 respondents (37\%) offered nonsurgical treatment (SBRT) for the metastasis.

\section{Conclusion}

Even in COVID-19 era, cancer remains top priority for treatment-timely treatment is vital. There is significant disruption to providing care to sarcoma patients with approximately half the respondents in our survey stated that chemotherapy and radiotherapy were no longer available for new patients. Almost all, emergency and most routine patients with high risk are being offered treatment. Wide variations exist on the use of PPE but might become more uniform as availability and cost improves. Telemedicine has made a big change. Palliative treatment is not being neglected. This survey brings to light changes of practice in most institutions, some of which are here to stay. Having picked up some good lessons from the modifications this pandemic has forced upon us, treatment of sarcomas post the pandemic might not be the same ever again. This survey will act as a reference point for tracking future trends in bone and soft tissue tumor management guidelines, as the COVID-19 scenario unfolds globally and particularly in India.

\section{Compliance with Ethical Standards}

Conflict of interest The authors declare that they have no conflict of interest.

\section{References}

1. Trapani, D., Marra, A., \& Curigliano, G. (2020). The experience on coronavirus disease 2019 and cancer from an oncology hub institution in Milan, Lombardy Region. European Journal of Cancer, 132, 199-206.

2. Weisel, K. C., Morgner-Miehlke, A., Petersen, C., Fiedler, W., Block, A., Schafhausen, P., et al. (2020). Implications of SARSCoV-2 infection and COVID-19 crisis on clinical cancer care: report of the University Cancer Center Hamburg. Oncology Research and Treatment, 43(6), 307-313.

3. Finley, C., Prashad, A., Camuso, N., Daly, C., Aprikian, A., Ball, C. G., et al. (2020). Guidance for management of cancer surgery during the COVID-19 pandemic. Canadian Journal of Surgery, 63(22), S2-S4.

4. Liang, W., Guan, W., Chen, R., Wang, W., Li, J., Xu, K., et al. (2020). Cancer patients in SARS-CoV-2 infection: a nationwide analysis in China. The Lancet Oncology, 21(3), 335-337.

5. André, N., Rouger-Gaudichon, J., Brethon, B., Phulpin, A., Thébault, É., Pertuisel, S., et al. (2020). COVID-19 in pediatric oncology from French pediatric oncology and hematology centers: High risk of severe forms? Pediatric Blood \& Cancer, 67(7), e28392.

6. Penel, N., Bonvalot, S., Minard, V., Orbach, D., Gouin, F., Corradini, N., et al. (2020). French Sarcoma Group proposals for management of sarcoma patients during the COVID-19 outbreak. Elsevier Ltd: Annals of Oncology.

7. Gulia, A., Singh, R., Pankaj, A., Panda, K., Raja, A., Tiwari, A., et al. (2020). Adapting management of sarcomas in COVID-19: An evidence-based review. The Indian Journal of Orthopaedics. https://doi.org/10.1007/s43465-020-00143-1.

8. Rivera, A., Ohri, N., Thomas, E., Miller, R., \& Knoll, M. A. (2020). The impact of COVID-19 on radiation oncology clinics and patients with cancer in the United States. Advances in Radiation Oncology. https://doi.org/10.1016/j.adro.2020.03.006.

9. Ministry of Health and Family Welfare Directorate General of Health Services [Emergency Medical Relief] Novel Coronavirus 
Disease 2019 (COVID-19): Guidelines on rational use of Personal Protective Equipment.

10. Cannistra, S. A., Haffty, B. G., \& Ballman, K. (2020). Challenges faced by medical journals during the COVID-19 pandemic. Journal of Clinical Oncology, 38(19), 2206-2207.

11. Hadaya, J., Schumm, M., \& Livingston, E. H. (2020). Testing individuals for coronavirus disease 2019 (COVID-19). JAMA, 323(19), 1981.
12. https://www.mohfw.gov.in/pdf/NotificationofICMguidelinesfor COVID19testinginprivatelaboratoriesiIndia.pdf

13. https://www.mohfw.gov.in/pdf/ICMRstrategyforCOVID19testingi nIndia.pdf

14. Knaul, F. M., Bhadelia, A., Rodriguez, N. M., Arreola-Ornelas, H., \& Zimmermann, C. (2018). The lancet commission on palliative care and pain relief-findings, recommendations, and future directions. The Lancet Global Health, 1(6), S5-S6. 